\title{
Gait Stability Has Phase-Dependent Dual-Task Costs in Parkinson's Disease
}

OPEN ACCESS

Edited by:

Emily Keshner,

Temple University, United States

Reviewed by:

Ruey-Meei WU,

National Taiwan University, Taiwan Pedro Ribeiro,

Universidade Federal do Rio de Janeiro, Brazil

*Correspondence: Peter C. Fino fino@ohsu.edu

Specialty section: This article was submitted to Movement Disorders, a section of the journal

Frontiers in Neurology

Received: 19 January 2018 Accepted: 07 May 2018

Published: 30 May 2018

Citation:

Fino PC, Mancini M, Curtze C, Nutt JG and Horak FB (2018)

Gait Stability Has Phase-

Dependent Dual-Task Costs

in Parkinson's Disease.

Front. Neurol. 9:373.

doi: 10.3389/fneur.2018.00373

\author{
Peter C. Fino ${ }^{1,2 *}$, Martina Mancini ${ }^{1,2}$, Carolin Curtze ${ }^{1}$, John G. Nutt ${ }^{1}$ and Fay B. Horak ${ }^{1,2}$ \\ ${ }^{1}$ Department of Neurology, Oregon Health \& Science University, Portland, OR, United States, ${ }^{2}$ Veterans Affairs Portland \\ Health Care System, Portland, OR, United States
}

Dual-task (DT) paradigms have been used in gait research to assess the automaticity of locomotion, particularly in people with Parkinson's disease (PD). In people with PD, reliance on cortical control during walking leads to greater interference between cognitive and locomotor tasks. Yet, recent studies have suggested that even healthy gait requires cognitive control, and that these cognitive contributions occur at specific phases of the gait cycle. Here, we examined whether changes in gait stability, elicited by simultaneous cognitive DTs, were specific to certain phases of the gait cycle in people with PD. Phasedependent local dynamic stability (LDS) was calculated for 95 subjects with PD and 50 healthy control subjects during both single task and DT gait at phases corresponding to (1) heel contact-weight transfer, (2) toe-off-early swing, and (3) single-support-mid swing. PD-related DT interference was evident only for the duration of late swing and LDS during the heel contact-weight transfer phase of gait. No PD-related DT costs were found in other traditional spatiotemporal gait parameters. These results suggest that PD-related DT interference occurs only during times where cortical activity is needed for planning and postural adjustments. These results challenge our understanding of DT costs while walking, particularly in people with PD, and encourage researchers to re-evaluate traditional concepts of DT interference.

Keywords: lyapunov exponents, locomotion, cognitive dual-task, local dynamic stability, dynamic postural control

\section{INTRODUCTION}

Locomotor deficits have been widely reported in people with Parkinson's disease (PD) due to the degeneration of basal ganglia and brainstem structures that contribute to control of gait and balance (1-5). To compensate for disrupted subcortical pathways, individuals with PD exhibit more goal-directed locomotion (6-8), with greater reliance on cortical networks when walking $(8,9)$. In particular, people with PD exhibit increased gait variability (10-13) and abnormal gait dynamics (i.e., how gait parameters vary over time) (14), often attributed to this loss of automaticity and increased cortical control of locomotion $(7,8,15)$.

The primary evidence for this compensatory cognitive control in people with PD stems from excessive dual-task (DT) cost $(16,17)$. DT paradigms involve a cognitive task performed concurrently with a locomotor task, producing interference between the tasks and leading to decreases in the performance of one or both tasks (18-20). While there are several prevailing theories to describe the nature of these performance deficits, called DT costs or dual-task changes (DTC) $(21,22)$, a common notion maintains that the regulation of the cognitive task and the regulation of gait 
interfere with one another. In healthy people, walking normally requires little cortical attention and therefore shows little DTCs, whereas in people with $\mathrm{PD}$, walking requires significant cortical compensation, resulting in large DTCs (18-20, 23, 24).

Larger DTCs in spatiotemporal measures such as stride time, stride length, and gait speed have been reported in people with PD compared to control subjects (25), and larger DTCs have been associated with PD severity $(19,26)$, or freezing of gait $(27,28)$. However, these spatiotemporal measures of gait do not separate specific phases within the gait cycle that may be critical to stable locomotion. For instance, electroencephalography studies have indicated that gait involves cortical contributions at specific phases to plan the next foot placement, transfer weight from one step to the next, and maintain stability (29-31). Therefore, the interference between the cognitive task demands and the compensatory cortical control of gait may be specific to certain phases of the gait cycle that depend most on cortical control.

To investigate whether people with PD have DTCs that are specific to certain phases of the gait cycle, we examined phase-dependent measures of gait stability and traditional spatiotemporal gait measures in subjects with idiopathic PD and healthy age-matched control subjects during self-paced, over-ground walking with and without a cognitive DT. Phasedependent local dynamic stability (LDS) of trunk movements was calculated at three phases of the gait cycle, corresponding to: (1) heel contact-weight transfer, (2) toe-off-early swing, and (3) single-support-mid swing. Phase-dependent LDS quantifies the rate at which local perturbations are attenuated during specific phases of the gait cycle (32). Previous studies have shown that phase-dependent LDS during weight transfer, but not other phases, is a sensitive predictor of falls in elderly populations and can differentiate gait in young and older adults, suggesting that dynamic stability during weight transfer, specifically, is sensitive to neuromotor changes related to fall risk due to aging (32-35).

Greater knowledge of how cognitive tasks interfere with locomotor demands in people with PD may facilitate targeted intervention strategies that focus on specific, highly affected components of gait. Therefore, the purpose of this study was to examine if people with PD exhibit phase-specific DTCs in local dynamic gait stability. We hypothesized that people with PD would exhibit the most severe DTC in dynamic stability during the weight transfer phase of gait compared with controls. We anticipated that these PD-related DTCs in dynamic stability associated with weight transfer would differ between subjects with PD and healthy control subjects while the DTCs in other spatiotemporal gait would not.

\section{MATERIALS AND METHODS}

\section{Participants}

As part of a larger study (Clinical Trials NCT02231073 and NCT02236286), 100 individuals with idiopathic PD were recruited for this baseline analysis. All subjects with PD had clinically diagnosed idiopathic PD by a neurologist and were tested in the practical OFF levodopa state, after withholding anti-parkinsonian medication for $\geq 12 \mathrm{~h}$. Inclusion criteria for subjects with PD were
(1) between 50 and 90 years old, (2) no major musculoskeletal or peripheral disorders (other than PD) that could significantly affect their balance and gait, (3) ability to stand and walk unassisted, and (4) met criteria for idiopathic PD according to the according to the Brain Bank Criteria for PD (36). In addition, 56 healthy elderly adults were recruited from the community. Exclusion criteria for both groups were as follows: any other neurological disorders or musculoskeletal impairments that interfere with gait or balance, and inability to follow instructions.

Five individuals with $\mathrm{PD}$ and six healthy controls were excluded from the final analysis due to technical considerations (see Analysis). Demographic characteristics for subjects retained in the final analysis for each group are provided in Table 1. This study was carried out in accordance with the recommendations of the Oregon Health \& Science University (OHSU) and Veterans Affairs Portland Health Care System (VAPORHCS) joint institutional review board (IRB) with written informed consent from all subjects. All subjects gave written informed consent in accordance with the Declaration of Helsinki. The protocol was approved by the OHSU (\#4131) and the OHSU/VAPORHCS joint IRB (\#8979).

\section{Procedures}

Subjects with PD were clinically rated by a trained examiner on the Motor Section (III) of the Unified PD Rating Scale (MDSUPDRS), which consists of 23 items related to bradykinesia, rigidity, tremor, and posture and gait signs rated on a four-point scale (37), prior to the mobility assessment. The Posture Instability and Gait Disability (PIGD) subscore was also calculated from the MDS-UPDRS Part III (38).

At the beginning of the mobility assessment, each participant performed a seated cognitive task of reciting every other letter of the alphabet for $1 \mathrm{~min}$. The number of total responses and the number of correct responses were recorded. Each participant was then outfitted with eight inertial sensors (APDM, Inc., Portland, OR, USA), worn on the sternum, lumbar spine, bilaterally on the wrists,

TABLE 1 | Demographic data.

\begin{tabular}{lccr}
\hline & Controls & PD & p-Value \\
\hline N & 50 & 95 & \\
\% Female & 38 & 32 & \\
Age (years) & $67.8(8.0)$ & $68.7(7.7)$ & 0.947 \\
Height (cm) & $171.7(9.8)$ & $174.2(10.2)$ & 0.176 \\
Mass (kg) & $73.8(14.6)$ & $79.5(15.2)$ & $\mathbf{0 . 0 3 3}$ \\
miniBEST & $24.6(2.2)$ & $18.4(4.7)$ & $<\mathbf{0 . 0 0 1}$ \\
TUG time (s) & $18.3(3.1)$ & $23.2(10.0)$ & $\mathbf{0 . 0 0 1}$ \\
MoCA & $26.8(1.9)$ & $25.5(3.6)$ & $\mathbf{0 . 0 1 9}$ \\
SCOPA-COG & $32.0(3.5)$ & $28.1(5.6)$ & $<\mathbf{0 . 0 0 1}$ \\
Fall in the past year (\%) & 12 & 38 & \\
Disease duration (years) & - & $7.0(5.2)$ & \\
MDS-UPDRS part III & - & $40.4(12.9)$ & \\
PIGD score & - & $5.0(3.2)$ & \\
H\&Y (range) & - & $2-3$ & \\
N with freezing of gait & - & 26 & \\
\end{tabular}

Where applicable, groups were compared using independent sample t-tests and a significance level of 0.05 .

Bold values indicate significant differences between $P D$ and control subjects. $P D$, Parkinson's disease; miniBEST, mini Balance Evaluation Systems Test; MoCA, Montreal Cognitive Assessment; PIGD, Posture Instability and Gait Disability. 
anterior distal region of the shanks, and feet. Each inertial sensor recorded tri-axial accelerations and angular velocities at $128 \mathrm{~Hz}$. Data from the wrist-sensors were not used for this study. As part of the larger study, participants completed several tests of balance and mobility, including the Timed Up and Go (TUG), mini Balance Evaluation Systems Test (miniBEST), and self-paced walking trials (Table 1). In addition, each participant completed the Montreal Cognitive Assessment (MoCA) (39) and SCOPA-COG.

Analysis of phase-dependent gait stability was based on two self-paced, walking trials: one 2-min trial with no added cognitive task [single-task (ST)] and one 1-min trial with a simultaneous cognitive task (DT). In both conditions, participants were instructed to walk at a comfortable pace back and forth continuously between two lines $7.62 \mathrm{~m}$ apart. In the ST condition, participants were instructed to walk for the entire $2 \mathrm{~min}$; no other task was given. In the DT condition, participants were instructed to walk for $1 \mathrm{~min}$ while reciting every other letter of the alphabet. The order of the conditions was not randomized; the ST condition was always completed before the DT condition. In the DT condition, participants were given no instruction regarding the prioritization of one task over the other. The number of correct responses during the DT condition was recorded.

\section{Analysis}

Raw 3-D accelerometer and gyroscope data were extracted from the sternum, lumbar spine, and shank inertial sensors for each walking trial. Each walking trial was segmented into multiple, straight walking bouts by removing turns and removing one stride immediately preceding and following each turn. Turns were identified using a threshold-based detection algorithm based on the axial angular velocity of the lumbar sensor (40). Heel-contact, toe-off, and mid-swing events were detected using the angular velocity of the shank as described by Salarian et al. (41). Each straight walking bout was then divided into non-overlapping segments of five consecutive, straight walking strides, with each stride timenormalized to 130 points to maintain equal data-length across segments. If a walking bout did not include at least five straight strides, it was excluded from the remainder of the analysis. Subjects were excluded entirely if they had no walking bouts with at least five consecutive straight strides in either the ST or DT conditions.

Phase-dependent LDS was calculated for each walking bout of five strides at three phases within the gait cycle, heel contactweight transfer; toe off-early swing; and single-support-mid swing, based on procedures described by Ihlen et al. (34). Briefly, a $6 \mathrm{D}$ state space $\boldsymbol{X}(t)=\left[a_{\mathrm{AP}}(t), a_{\mathrm{ML}}(t), a_{\mathrm{ML}}(t), \omega_{\mathrm{AP}}(t), \omega_{\mathrm{ML}}(t), \omega_{\mathrm{VT}}(t)\right]$ was constructed using the $3 \mathrm{D}$ trunk accelerations $a(t)$ and $3 \mathrm{D}$ trunk angular velocities $\omega(t)$ from the sternum inertial sensor. Next, points corresponding to heel-contact, toe-off, and midswing events were found within the state space, and two nearest neighbors within the space were identified for each event. For each gait event, the average distances from the trajectories of the two nearest neighbors to the trajectory of gait event were tracked for one step, and mean log divergence curves were created by mapping the average distance across all similar gait events (e.g., all heel-contact events, all toe-off events, and all mid-swing events) as a function of the percentage of normalized stride.
Phase-dependent LDS was then estimated for each segment using maximum finite-time Lyapunov exponents calculated from the slope of the mean log divergence curves from the initial gait event to the next $10 \%$ of the step cycle (i.e., $5 \%$ of the gait cycle) for each phase, heel contact $\left(\lambda_{\mathrm{HC}}\right)$, toe off $\left(\lambda_{\mathrm{TO}}\right)$, and mid swing $\left(\lambda_{\mathrm{MS}}\right)$ (e.g., heel contact $+5 \%$ of gait cycle, toe off $+5 \%$ of gait cycle, and mid swing $+5 \%$ of the gait cycle, respectively). This procedure can be described mathematically using the following equation:

$$
\lambda_{\text {bout }}=\frac{\left\langle\ln \left\langle d_{i}(t)\right\rangle\right\rangle_{\text {step }}}{t},
$$

where $\left\langle d_{i}(t)\right\rangle$ is the average Euclidean distance between the $i$ nearest neighbor trajectories and the reference trajectory at each point in time $t$, where the gait event (e.g., heel contact, toe off, or mid swing) defined $t=0$ within the state space, $\langle\ldots\rangle_{\text {step }}$ is the average over all steps within the bout, and $\lambda_{\text {bout }}$ is the estimate of phase-dependent LDS for a single bout. The median $\lambda_{\mathrm{HC}}, \lambda_{\mathrm{TO}}$, and $\lambda_{\mathrm{MS}}$ across all walking bouts was used as the final estimate of phase-dependent LDS at heel contact, toe off, and mid swing, respectively.

Traditional LDS, $\lambda_{\text {Kantz }}$, was also calculated for each walking segment of five time-normalized strides following Kantz's algorithm (42) and previous reports for estimating local dynamics stability over short bouts of gait (43-45). A 9D state space was constructed from the three-dimensional trunk accelerations and their twice time-delayed copies using a fixed time delay of 0.25 of the average stride time. For each point, the average distance to the two nearest neighbors of the trajectory were tracked for one step, and mean log divergence curves were created by mapping the average distance across all points as a function of the percentage of normalized stride. Traditional LDS, $\lambda_{\text {Kantz }}$, was then estimated for each segment using maximum finite-time Lyapunov exponents calculated from the slope of the mean log divergence curves from the 0 to 0.5 strides, and the median across all walking segments was used as the final estimate of $\lambda_{\text {Kantz. }}$. For all four stability outcomes, greater values of $\lambda$ indicate faster divergence or nearby trajectories in state space and are therefore associated with less stability; smaller values of $\lambda$ indicate slower divergence and are typically associated with increased stability $(46,47)$.

To compare the DTC of stability outcomes to the DTC of traditional gait measures, temporal gait measures of stride time, double support time, early swing time (toe off to mid swing), and late swing time (mid swing to heel contact) were calculated from the difference in time between respective gait events. Gait speed and stride length were calculated from Mobility Lab software using analysis version 3.0 (Mobility Lab v2, APDM, Inc., Portland, OR, USA).

To evaluate the performance on the cognitive task, the total number of responses and the number of correct responses were tabulated for both the seated and DT walking conditions. Accuracy was calculated as the number of correct responses divided by the total number of responses $\left(\right.$ Accuracy $\left.=\frac{\# \text { Correct }}{\text { Total }}\right)$. For cognitive task outcomes of total responses, correct responses, and accuracy, the DTC was calculated as the change relative to seated. 


\section{Statistical Analysis}

Independent sample $t$-tests compared age, height, mass, miniBEST scores, MoCA scores, and SCOPA-COG scores between the $\mathrm{PD}$ and control groups. To investigate whether outcomes differed between groups, linear mixed models were fit for each stability outcome ( $\lambda_{\mathrm{HC}}, \lambda_{\mathrm{TO}}, \lambda_{\mathrm{MS}}$, and $\left.\lambda_{\text {Kantz }}\right)$, spatiotemporal measure of gait (gait speed, stride length, stride time, double support time, early swing time, and late swing time), and cognitive task outcome (total responses, correct responses, and accuracy). Each model was adjusted for group, task (ST versus DT), and the group ${ }^{\star}$ task interaction. The group ${ }^{\star}$ task interaction term was included in each model to test whether groups had different linear DTC between task conditions. Each model included a random intercept for each subject to account for the repeated measurements within each subject. For the cognitive outcomes, the task effect compared seated to walking conditions. Gait speed was included as a covariate in models for stability outcomes to account for variations in stability with gait speed $(48,49)$.

To confirm that any significant group ${ }^{\star}$ task interaction was robust to methods of calculating DTC (23), we performed post hoc analyses on any outcome with a significant group ${ }^{\star}$ task interaction. As the group ${ }^{*}$ task interaction term in the linear mixed models assessed the linear DTC between tasks (DT - ST), group differences in the DTC as a percentage $\left(\% \mathrm{DTC}=\frac{\mathrm{DT}-\mathrm{ST}}{\mathrm{ST}} \times 100 \%\right)$ were tested using independent sample $t$-tests. To limit the number of comparisons, the comparison of \%DTC between groups was only performed on outcome measures with a significant group ${ }^{\star}$ task interaction.

To assess whether DTCs were associated with disease duration, severity, or cognitive function in PD, Spearman correlation coefficients compared the \%DTCs of each outcome with a significant group ${ }^{\star}$ task interaction to disease duration, the MDS-UPDRS Part III subscore, the PIGD score from the MDS-UPDRS, the miniBEST score, the MoCA score, and the SCOPA-COG score. All statistical analysis was performed in MATLAB r2017a (The Mathworks Inc., Natick, MA, USA) using the Statistics and Machine Learning Toolbox. A significance level of 0.05 was used throughout.

\section{RESULTS}

Ninety-five subjects with PD and 50 healthy control subjects were retained in the final analysis after excluding five subjects with PD and six control subjects with no bouts of at least five strides during both ST and DT gait. The PD and control groups had medians (IQR) of 12 (2) and 14 (3) bouts of ST gait, respectively, and 6 (1) and 6 (2) bouts of DT gait, respectively, included in the analysis. There were no significant differences between groups in age or height. The PD group had significantly greater mass, lower miniBEST, MoCA, and SCOPA-COG scores, and had slower TUG times (Table 1). Univariate descriptive statistics for each outcome are shown in Table 2.

A significant group ${ }^{*}$ task effect was found for phase-dependent stability at weight transfer $\left(\lambda_{\mathrm{HC}}\right)$ meaning that subjects with PD became less dynamically stable in the DT condition relative to the difference between conditions in the control subjects (Table 3, Figure 1). No group ${ }^{\star}$ task effect was found for stability at other phases $\left(\lambda_{\mathrm{TO}}, \lambda_{\mathrm{MS}}\right)$ or when assessed without regards to phase $\left(\lambda_{\text {Kantz }}\right)$. Phase-dependent stability at weight transfer $\left(\lambda_{\mathrm{HC}}\right)$ and mid swing $\left(\lambda_{\mathrm{MS}}\right)$ was significantly greater (i.e., less stable) with faster gait speeds, while non-phase-dependent stability $\left(\lambda_{\text {Kantz }}\right)$ was significantly lower (i.e., more stable) with faster gait.

A significant group task interaction effect was found for time spent in the late swing phase, where, relative to the ST condition, control subjects increased the time spent in late swing in the DT condition but subjects with PD did not change. No other spatiotemporal measure had a significant

TABLE 2 | Univariate means (SD) of each outcome stratified by group and condition.

\begin{tabular}{|c|c|c|c|c|}
\hline & \multicolumn{2}{|c|}{ Single task } & \multicolumn{2}{|c|}{ Dual task } \\
\hline & Mean & SD & Mean & SD \\
\hline \multicolumn{5}{|c|}{ Gait speed (m/s) } \\
\hline Control & 1.12 & 0.14 & 0.95 & 0.17 \\
\hline PD & 0.93 & 0.20 & 0.78 & 0.19 \\
\hline \multicolumn{5}{|c|}{ Stride length (m) } \\
\hline Control & 1.19 & 0.08 & 1.11 & 0.10 \\
\hline PD & 0.99 & 0.19 & 0.89 & 0.19 \\
\hline \multicolumn{5}{|c|}{ Stride time (s) } \\
\hline Control & 1.04 & 0.13 & 1.07 & 0.15 \\
\hline PD & 1.07 & 0.16 & 1.09 & 0.15 \\
\hline \multicolumn{5}{|c|}{ Time in double support (\%) } \\
\hline Control & 22.5 & 3.9 & 24.6 & 3.9 \\
\hline PD & 23.9 & 4.8 & 26.7 & 6.2 \\
\hline \multicolumn{5}{|c|}{ Time in early swing (\%) } \\
\hline Control & 51.2 & 4.8 & 48.1 & 5.4 \\
\hline PD & 49.2 & 5.6 & 46.4 & 6.4 \\
\hline \multicolumn{5}{|c|}{ Time in late swing (\%) } \\
\hline Control & 26.5 & 3.3 & 27.4 & 3.3 \\
\hline PD & 27.0 & 3.3 & 27.0 & 3.7 \\
\hline \multicolumn{5}{|c|}{ Total cognitive responses $(n)^{\mathrm{a}}$} \\
\hline Control & 36 & 7 & 35 & 8 \\
\hline PD & 32 & 10 & 29 & 8 \\
\hline \multicolumn{5}{|c|}{ Correct cognitive responses $(n)^{\mathrm{a}}$} \\
\hline Control & 34 & 8 & 31 & 8 \\
\hline PD & 29 & 11 & 26 & 9 \\
\hline \multicolumn{5}{|c|}{ Cognitive task accuracy $(\%)^{a}$} \\
\hline Control & 93 & 9 & 89 & 10 \\
\hline PD & 90 & 11 & 88 & 11 \\
\hline \multicolumn{5}{|l|}{$\lambda_{\mathrm{HC}}$} \\
\hline Control & 0.15 & 0.04 & 0.13 & 0.04 \\
\hline PD & 0.13 & 0.04 & 0.12 & 0.04 \\
\hline \multicolumn{5}{|l|}{$\lambda_{\text {Tо }}$} \\
\hline Control & 0.07 & 0.02 & 0.08 & 0.02 \\
\hline PD & 0.08 & 0.02 & 0.08 & 0.02 \\
\hline \multicolumn{5}{|l|}{$\lambda_{\mathrm{MS}}$} \\
\hline Control & 0.11 & 0.02 & 0.11 & 0.02 \\
\hline PD & 0.12 & 0.02 & 0.11 & 0.02 \\
\hline \multicolumn{5}{|l|}{$\lambda_{\text {Kantz }}$} \\
\hline Control & 0.30 & 0.07 & 0.34 & 0.08 \\
\hline PD & 0.35 & 0.07 & 0.37 & 0.09 \\
\hline
\end{tabular}

aST condition for cognitive responses refers to the seated condition. $P D$, Parkinson's disease. 
TABLE 3 | Results from the linear mixed models for each stability measure.

\begin{tabular}{|c|c|c|c|c|c|}
\hline & Beta & SE & Lower Cl & Upper Cl & $p$-Value \\
\hline \multicolumn{6}{|l|}{$\lambda_{\mathrm{HC}}$} \\
\hline Intercept & 0.072 & 0.015 & 0.042 & 0.103 & $<0.001$ \\
\hline Task (ref ST) & -0.011 & 0.005 & -0.020 & -0.001 & 0.024 \\
\hline Gait speed & 0.070 & 0.013 & 0.045 & 0.096 & $<0.001$ \\
\hline Group (ref controls) & -0.006 & 0.007 & -0.019 & 0.007 & 0.390 \\
\hline Group`Task & 0.011 & 0.005 & 0.000 & 0.021 & 0.043 \\
\hline \multicolumn{6}{|l|}{$\lambda_{\text {TO }}$} \\
\hline Intercept & 0.077 & 0.009 & 0.060 & 0.094 & $<0.001$ \\
\hline Task (ref ST) & 0.001 & 0.003 & -0.006 & 0.008 & 0.756 \\
\hline Gait speed & -0.003 & 0.007 & -0.017 & 0.011 & 0.681 \\
\hline Group (ref controls) & 0.008 & 0.004 & 0.001 & 0.012 & 0.029 \\
\hline Group^Task & -0.003 & 0.004 & -0.011 & 0.004 & 0.412 \\
\hline \multicolumn{6}{|l|}{$\lambda_{\mathrm{MS}}$} \\
\hline Intercept & 0.072 & 0.009 & 0.054 & 0.089 & $<0.001$ \\
\hline Task (ref ST) & 0.004 & 0.003 & -0.002 & 0.010 & 0.163 \\
\hline Gait speed & 0.031 & 0.008 & 0.016 & 0.046 & $<0.001$ \\
\hline Group (ref controls) & 0.015 & 0.004 & 0.008 & 0.023 & $<0.001$ \\
\hline Group^Task & -0.005 & 0.003 & -0.011 & 0.002 & 0.178 \\
\hline \multicolumn{6}{|l|}{$\lambda_{\text {Kantz }}$} \\
\hline Intercept & 0.471 & 0.031 & 0.410 & 0.533 & 0.000 \\
\hline Task (ref ST) & 0.009 & 0.013 & -0.017 & 0.035 & 0.486 \\
\hline Gait speed & -0.151 & 0.026 & -0.203 & -0.100 & $<0.001$ \\
\hline Group (ref controls) & 0.020 & 0.014 & -0.007 & 0.048 & 0.142 \\
\hline Group^Task & -0.011 & 0.015 & -0.041 & 0.018 & 0.452 \\
\hline
\end{tabular}

Lower and upper 95\% confidence intervals (Cl) for beta are also presented. Bold values indicate significant effects at $p<0.05$.

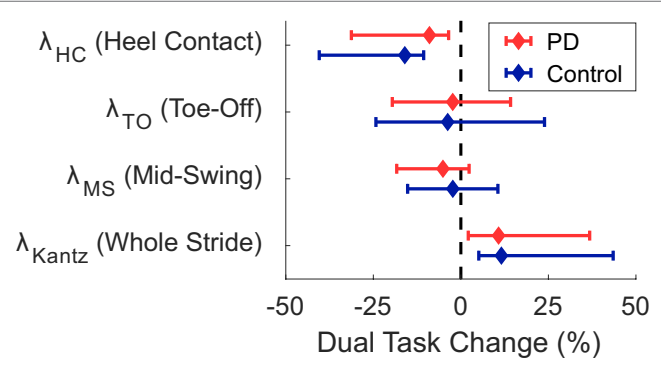

FIGURE 1 | Median dual-task changes (DTC) as percentages (\%DTC) and IQRs for phase-dependent local dynamic stability (LDS) measures calculated at (1) heel contact - weight transfer, $\lambda_{\text {HC }}$, (2) toe off-early swing, $\lambda_{\text {TO }}$, and (3) single-support-mid swing, $\lambda_{\mathrm{Ms}}$, and traditional LDS calculated at all points within a stride, $\lambda_{\text {Kantz. }}$. DTCs were calculated as a percentage change with respect to single-task gait. *Phase-dependent LDS during heel contactweight transfer, $\lambda_{H C}$, was significantly different between groups whether calculated as a linear change (see Table 3 ), or as a percentage change. Group differences in DTCs as percentages were not tested on other stability outcomes as the group*task interactions were not significant in the initial linear mixed models.

group ${ }^{\star}$ task interaction effect indicative of PD-related DTCs. Subjects with PD had significantly slower gait speed, shorter stride lengths, and spent less time in early swing compared to controls (Table 4; Figure 2). The DT condition led to slower gait speeds, shorter stride lengths, more time spent in double support, and less time in early swing compared to the ST condition across all subjects.
TABLE 4 | Results from the linear mixed models for each spatiotemporal measure.

\begin{tabular}{|c|c|c|c|c|c|}
\hline & Beta & SE & Lower $\mathrm{Cl}$ & Upper Cl & $p$-Value \\
\hline \multicolumn{6}{|l|}{ Gait speed (m/s) } \\
\hline Intercept & 1.12 & 0.03 & 1.07 & 1.17 & $<0.001$ \\
\hline Task (ref ST) & -0.17 & 0.02 & -0.20 & -0.14 & $<0.001$ \\
\hline Group (ref controls) & -0.20 & 0.03 & -0.26 & -0.13 & $<0.001$ \\
\hline Group*Task & 0.02 & 0.02 & -0.02 & 0.06 & 0.368 \\
\hline \multicolumn{6}{|l|}{ Stride length (m) } \\
\hline Intercept & 1.19 & 0.02 & 1.15 & 1.24 & $<0.001$ \\
\hline Task (ref ST) & -0.08 & 0.01 & -0.11 & -0.06 & $<0.001$ \\
\hline Group (ref controls) & -0.20 & 0.03 & -0.26 & -0.14 & $<0.001$ \\
\hline Group*Task & -0.02 & 0.01 & -0.05 & 0.00 & 0.090 \\
\hline \multicolumn{6}{|l|}{ Stride time (s) } \\
\hline Intercept & 1.04 & 0.02 & 0.99 & 1.08 & $<0.001$ \\
\hline Task (ref ST) & 0.03 & 0.03 & -0.02 & 0.08 & 0.230 \\
\hline Group (ref controls) & 0.04 & 0.03 & -0.02 & 0.09 & 0.157 \\
\hline Group*Task & -0.02 & 0.03 & -0.08 & 0.04 & 0.532 \\
\hline \multicolumn{6}{|c|}{ Time in double support (\%) } \\
\hline Intercept & 22.5 & 0.7 & 21.1 & 23.9 & $<0.001$ \\
\hline Task (ref ST) & 2.1 & 0.4 & 1.3 & 3.0 & $<0.001$ \\
\hline Group (ref controls) & 1.4 & 0.9 & -0.3 & 3.1 & 0.107 \\
\hline Group*Task & 0.6 & 0.5 & -0.4 & 1.7 & 0.231 \\
\hline \multicolumn{6}{|c|}{ Time in early swing (\%) } \\
\hline Intercept & 51.1 & 0.8 & 49.6 & 52.7 & $<0.001$ \\
\hline Task (ref ST) & -3.0 & 0.5 & -4.0 & -2.0 & $<0.001$ \\
\hline Group (ref controls) & -2.0 & 1.0 & -3.9 & -0.0 & 0.048 \\
\hline Group`Task & -0.2 & 0.6 & -0.9 & 1.4 & 0.732 \\
\hline \multicolumn{6}{|l|}{ Time in late swing (\%) } \\
\hline Intercept & 26.5 & 0.5 & 25.6 & 27.5 & $<0.001$ \\
\hline Task (ref ST) & 0.9 & 0.3 & 0.3 & 1.4 & 0.002 \\
\hline Group (ref controls) & -0.5 & 0.6 & -0.7 & 1.6 & 0.431 \\
\hline Group*Task & -0.9 & 0.3 & -1.5 & -0.2 & 0.010 \\
\hline
\end{tabular}

Lower and upper 95\% confidence intervals (Cl) for beta are also presented. Bold values indicate significant effects at $p<0.05$.

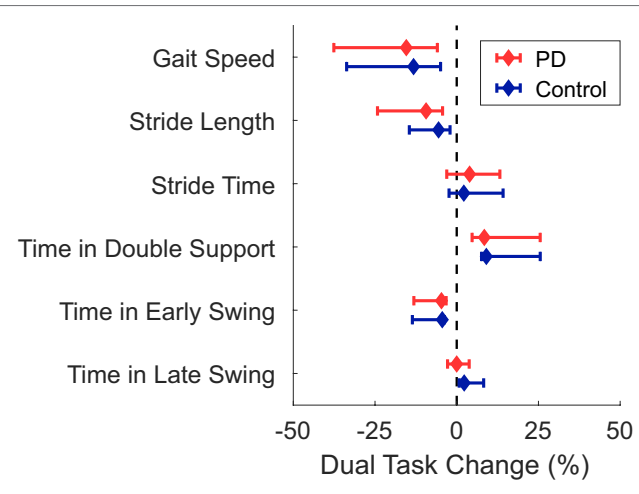

FIGURE 2 | Median dual-task changes (DTC) as percentages (\%DTC) and IQRs for spatiotemporal measures of gait for people with Parkinson's disease (PD) (red) and healthy control subjects (blue). DTCs were calculated as a percentage change with respect to single-task gait. ${ }^{*}$ Time spent in late swing was significantly different between groups whether calculated as a linear change (see Table 4), or as a percentage change. Group differences in DTCs as percentages were not tested on other spatiotemporal outcomes as the group*task interactions were not significant in the initial linear mixed models.

There was no significant group ${ }^{\star}$ task interaction for any cognitive task outcome (Table 5; Figure 3). Main effects of group and task were found for the number of correct responses, but not for 
TABLE 5 | Results from the linear mixed models for measures of cognitive task performance.

\begin{tabular}{lcccrr}
\hline & Beta & SE & Lower Cl & Upper Cl & p-Value \\
\hline Number of total responses & & & & \\
Intercept & 35.99 & 1.23 & 33.45 & 38.30 & $<0.001$ \\
Task (ref ST) & -1.30 & 0.79 & -2.86 & 0.26 & 0.103 \\
Group (ref controls) & $-\mathbf{3 . 8 6}$ & $\mathbf{1 . 5 3}$ & $-\mathbf{6 . 8 7}$ & $-\mathbf{0 . 8 6}$ & $\mathbf{0 . 0 1 2}$ \\
Group*Task & -1.07 & 1.00 & -3.00 & 0.92 & 0.299 \\
Number of correct responses & & & & \\
Intercept & 33.5 & 1.29 & 30.96 & 36.04 & $<0.001$ \\
Task (ref seated) & $\mathbf{- 2 . 6 0}$ & $\mathbf{0 . 8 1}$ & $-\mathbf{4 . 1 9}$ & $-\mathbf{1 . 0 2}$ & $\mathbf{0 . 0 0 1}$ \\
Group (ref controls) & $\mathbf{- 4 . 2 9}$ & $\mathbf{1 . 6 0}$ & $\mathbf{- 7 . 4 5}$ & $\mathbf{- 1 . 1 4}$ & $\mathbf{0 . 0 0 8}$ \\
Group*Task & -0.29 & 1.01 & -2.28 & 1.69 & 0.771 \\
Cognitive task accuracy (\%) & & & & \\
Intercept & 92.5 & 14.7 & 89.6 & 95.4 & $<0.001$ \\
Task (ref seated) & $\mathbf{- 3 . 4}$ & $\mathbf{1 . 3}$ & $\mathbf{- 5 . 9}$ & $-\mathbf{0 . 8}$ & $\mathbf{0 . 0 1 0}$ \\
Group (ref controls) & -2.6 & 1.8 & -6.2 & 0.9 & 0.149 \\
Group*Task & -1.4 & 1.6 & -1.8 & 4.6 & 0.399 \\
\hline
\end{tabular}

Lower and upper 95\% confidence intervals (Cl) for beta are also presented. Bold values indicate significant effects at $p<0.05$.

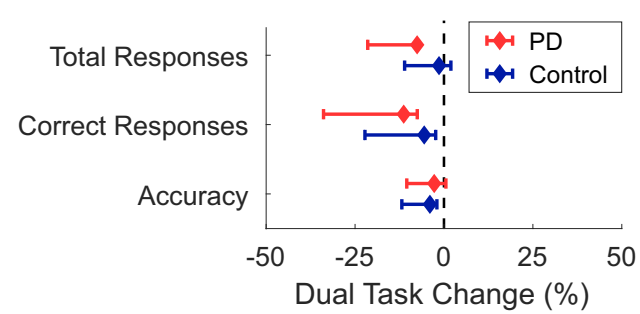

FIGURE 3 | Median dual-task changes (DTC) as percentages (\%DTC) and IQRs for cognitive outcomes for people with Parkinson's disease (PD) (red) and healthy control subjects (blue). DTCs were calculated as a percentage change with respect to seated. Group differences in DTCs as percentages were not tested for any cognitive outcome as no group*task interactions were significant in the initial linear mixed models.

the number of total responses or accuracy. Subjects with PD had fewer correct responses than the control group across both conditions, and the walking condition had fewer correct responses than the seated condition across both groups.

Post hoc $t$-tests were only performed on the \%DTC for two outcomes, $\lambda_{\mathrm{HC}}$ and time spent in late swing, as those were the only outcomes with significant group ${ }^{\star}$ task interactions in the linear mixed models. The $\% \mathrm{DTC}$ for $\lambda_{\mathrm{HC}}$ was significantly smaller in subjects with PD compared with controls $(t=-2.56, p=0.012)$. Similarly, the \%DTC for time spent in late swing was significantly smaller in subjects with PD compared to controls $(t=-2.78$, $p=0.006)$.

The $\%$ DTC for time in late swing was significantly associated with TUG time in controls only $(\rho=0.41, p=0.004)$, but not in subjects with PD. The \%DTCs of $\lambda_{\mathrm{HC}}$ and time in late swing were not significantly associated with disease duration, MDS-UPDRS Part III subscore, or UPDRS PIGD score in subjects with PD (Table 6). The \%DTCs of $\lambda_{\mathrm{HC}}$ and time in late swing were not associated with miniBEST, MoCA, or SCOPA-COG scores, or with age, height, or mass in either group (Figure 4).
TABLE 6 | Spearman correlation coefficients and $p$-values for comparisons between the \%DTC of $\lambda_{\mathrm{HC}}$ and clinical characteristics in subjects with Parkinson's disease.

\begin{tabular}{|c|c|c|c|c|c|c|}
\hline & \multicolumn{2}{|c|}{ Disease duration } & \multicolumn{2}{|c|}{$\begin{array}{c}\text { MDS-UPDRS } \\
\text { part III }\end{array}$} & \multicolumn{2}{|c|}{$\begin{array}{c}\text { Posture } \\
\text { Instability and } \\
\text { Gait Disability }\end{array}$} \\
\hline & $\rho$ & $p$-Value & $\rho$ & $p$-Value & $\rho$ & $p$-Value \\
\hline$\%$ DTC $\lambda_{\mathrm{HC}}$ & -0.011 & 0.917 & 0.091 & 0.384 & 0.063 & 0.549 \\
\hline $\begin{array}{l}\text { \%DTC time in late } \\
\text { swing }\end{array}$ & 0.039 & 0.709 & 0.075 & 0.475 & 0.109 & 0.294 \\
\hline
\end{tabular}

\section{DISCUSSION}

We compared the DTC on phase-dependent LDS during phases of the gait cycle beginning with heel contact, toe off, and mid swing in people with PD and healthy matched controls. Compared to controls, people with PD only demonstrated greater DTCs during the phase beginning at heel contact and corresponding to the weight transfer phase of gait. Many previous studies have described larger DTCs on spatiotemporal measures of gait in people with PD compared to controls [see review by Kelly et al. (25)], but these measures cannot examine intra-stride patterns. Our results suggest that cognitive DTs affect gait stability in an intra-stride, phase-specific pattern in people with $\mathrm{PD}$.

Increasing evidence has suggested that gait has intermittent contributions from the cortex, and that these contributions are locked to specific phases of the gait cycle $(29,31,50-52)$. Cortical activity in the premotor cortex is highest during single-limb stance prior to heel contact, representative of cortical planning of balance control and foot placement $(30,50)$. Others have reported elevated activity in the sensorimotor cortices during weight transfer $(31,52,53)$, indicative of sensory feedback for state estimation of postural adjustments (54). While it is not clear how variations in cortical activity affect DT interference, our results suggest DTC can similarly fluctuate within a gait cycle.

We found significant DTCs, indicated by a significant main effect of task, in both the PD and control groups with slower gait speeds, shorter stride lengths, more time spent in double support, and less time spent in early swing compared to the ST condition. However, these DTCs did not differ between subjects with PD and healthy control subjects. Previous work by Rochester et al. (23) suggested that DT deficits in PD stem from two underlying causes: age-related DT declines in overall gait performance and PD-related DT deficits in specific measures of postural control. Specifically, PD-related DT deficits were apparent only in step width and step width variability (23), implying that cognitive tasks only have PD-related interference with measures pertaining to the unstable mediolateral (ML) direction during gait $(55,56)$. Stable gait is largely achieved by placing the swing limb to redirect the lateral movement of the center-of-mass $(57,58)$. While weight transfer occurs after placement of the swing limb, planning the placement of the swing limb occurs during second half of the swing phase (59), during a period of elevated activity 

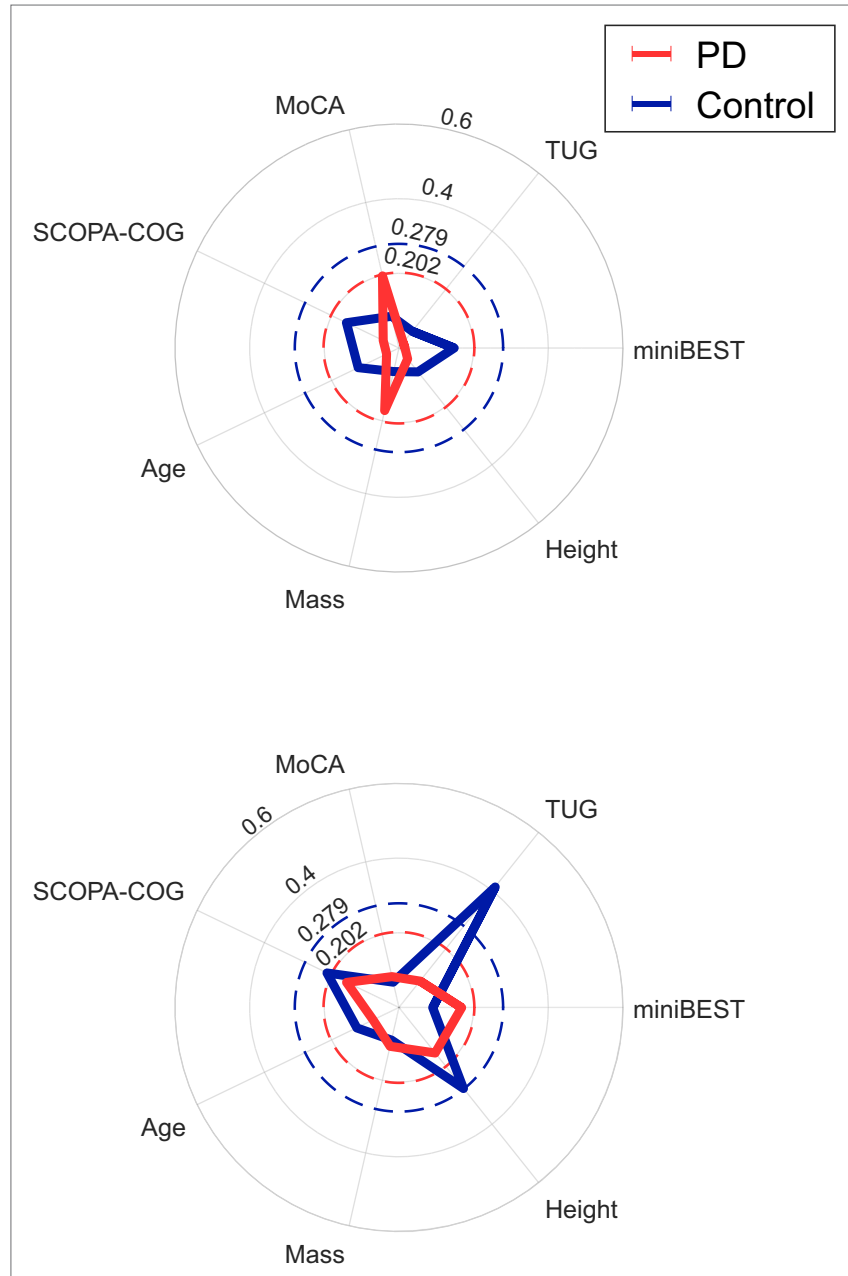

FIGURE 4 | Radar plots of the absolute value of Spearman's correlation coefficients between demographic and clinical outcomes and the \%DTCs of $\lambda_{\text {HC }}$ (top) or time in late swing (bottom). The dashed red and blue circles indicate the critical $\rho$ value for $p=0.05$ for Parkinson's disease (PD) and control groups, respectively. The \%DTC for time in late swing was significantly associated with TUG time in controls only ( $\rho=0.41, p=0.004)$. There were no other significant correlations $(p>0.05)$.

in the premotor cortex (50). In a study of healthy elderly, Bruijn et al. (30) found that stabilizing healthy young participants in the ML direction significantly decreased step width, improved trunk stability, and reduced the activity in the premotor cortex immediately before and during weight transfer. Therefore, it appears that stability, particularly in the ML direction, might require significant activity from the premotor and supplementary motor areas (SMA) for correct limb placement and weight transfer. Thus, the PD-related DTCs specific to the duration of the late swing phase of gait and gait stability during weight transfer are consistent with the effects of basal ganglia degeneration on SMA connectivity and postural adjustments in people with PD (24, 60-62). While we lack data on cortical activation to make firm conclusions about the nature of the DTC-related deficits we observed, we speculate that the PD-related DTCs during the late swing phase and weight transfer $\left(\lambda_{\mathrm{HC}}\right)$ may be indicative of greater cortical involvement for locomotion in $\mathrm{PD}$, due to reduced automaticity $(7,8)$. In people with $\mathrm{PD}$, reduced automaticity and increased cortical control over action has been put forward to explain DT costs during gait (23). Our results suggest that DT interference, possibly due to increased reliance on cortical control in $\mathrm{PD}$, may be more likely to occur at specific phases of gait that normally require cortical activity for stabilization, such as during late swing and weight transfer, as there may be less cognitive resources available for concurrent tasks at these phases.

We compared several spatiotemporal measures and measures of stability, but only phase-dependent LDS at weight transfer $\left(\lambda_{\mathrm{HC}}\right)$, and the time spent in late swing immediately prior to weight transfer, demonstrated PD-related DTCs. This result agrees with Rochester et al. (23), who similarly found differences in postural stability measures of step width, but failed to find PD-related differences in step length, step time, or step velocity. Furthermore, these results suggest that DT assessments may ignore temporal variation in the demands of the locomotor task. While several models of DT interference exist (e.g., bottleneck, resource limiting, and cross talk) (21), an implicit assumption across all models is that the two competing tasks occur simultaneously and uniformly. While studies have investigated how different cognitive tasks with variations in attentional focus over time influence DT costs during gait (63-65), few studies have examined the temporal variation of the demands of the locomotor task. Nonetheless, the idea that attentional demands vary across a gait cycle was suggested early on by Lajoie et al. (66), who found verbal reaction time was slower during single support compared to double support phases of the gait cycle. However, while Lajoie et al. (66), found reaction time varied by gait cycle in healthy young adults, they did not find DT differences in gait variables when assessing entire strides such as cadence, stride length, stride time, and gait speed. The general lack of consideration for intra-stride changes related to the locomotor task may help explain why DT assessments of gait have little added value over ST assessments when predicting future falls (67, 68). Supporting this notion, we found PD-related, DT interference on LDS only at a specific phase of gait, weight transfer. However, it is possible that severe PD-related DTCs, even if occurring only around weight transfer, could manifest in spatiotemporal measures of whole strides. Other studies have found PD-related DTCs in a variety of spatiotemporal measures, but there is variability about the magnitude of the effect and which spatiotemporal measures are affected (25). A phase-specific DTC in people with PD may explain some of this variability, where the PD-related DTC is blurred across the entire stride and only large magnitude DTCs are measurable. Combined, these results suggest that assessments should examine specific phases of gait, and that targeted interventions should specifically focus on improving the automaticity of foot placement and weight transfer during gait.

Few, if any, studies have compared phase-dependent LDS between people with PD and healthy controls. Yet, our results agree with previous studies that found phase-dependent LDS differences between young and older adults (33) and elderly fallers and non-fallers (34) specific to the weight transfer phase. 
Notably, older adults had larger $\lambda_{\mathrm{HC}}$, indicative of less stable dynamics, than young adults during steady-state treadmill walking (33). In a later analysis of data obtained during uncontrolled walking, Ihlen and colleagues $(34,35)$ found elderly with a history of falls had lower $\lambda_{\mathrm{HC}}$ values than non-fallers during daily living activities, indicating more stable dynamics. This disparity was attributed to fallers engaging in less complex tasks at home. A separate analysis found $\lambda_{\mathrm{HC}}$ increased with increasing gait speed (32), suggesting gait speed may have played a role in the lower $\lambda_{\mathrm{HC}}$ values in fallers compared to non-fallers. In our study, the control subjects decreased $\lambda_{\text {HC }}$ and slowed down when walking with a cognitive task, while individuals with PD slowed down but did not proportionally change $\lambda_{\text {HC. Therefore, }}$ after adjusting for changes in gait speed, our results can be interpreted similarly to the previous studies on steady-state gait and aligns with the larger body of literature on LDS, where larger $\lambda$ values indicate less stability (47). Accordingly, cognitive tasks during gait induced less stable dynamics during weight transfer in people with PD compared with similar-aged, elderly control subjects.

While this study benefited from a large sample size, several limitations should be considered when generalizing the results. First, the analysis of LDS and phase-dependent LDS was performed on a small number of consecutive strides. The small number of stride may have increased the within-subject variability across bouts which was partially mitigated by obtaining many bouts of gait (44). In preliminary analyses, we excluded 26 subjects with PD and 16 control subjects who had less than four bouts-21\% of the current sample-and we found identical results as presented here, suggesting that the current results are robust; the results do not appear to be driven by subjects with a small number of bouts. However, the present conclusions could be strengthened in future analyses considering a greater number of, and longer, bouts of consecutive strides.

Second, all subjects performed the same cognitive task, which introduced two confounding variables: between-subject differences in cognition and temporal variations in cognitive load as mentioned earlier. The PD group had significantly fewer correct responses across both seated and walking tasks, despite similar total responses, suggesting that our results may be associated with cognitive differences between groups. Yet, the DTC of $\lambda_{\mathrm{HC}}$ was not associated with the MoCA or the SCOPA-COG within either group, suggesting that cognitive differences alone do not explain our results. Furthermore, the lack of a significant group ${ }^{*}$ task interaction for any of the cognitive outcomes suggests that the PD group did not prioritize the cognitive and motor tasks differently than controls. It is possible the fixed order of the conditions may have introduced an order effect. However, the order was consistent across groups and the primary inferences were drawn from the group ${ }^{\star}$ task interaction. Similarly, the difference in duration between the walking conditions ( 2 min ST versus 1 min DT) led to fewer strides and bouts within the DT condition. The shorter DT duration was selected to accommodate people with PD who had difficulty completing 2-min of continuous DT walking. It is possible the different durations influenced the main effect of task, but it is unlikely the main inferences drawn from the group ${ }^{\star}$ task interaction were affected.

Finally, it is unclear how freezing of gait influenced our results. While bouts of gait that included a freezing episode were excluded from any analysis, it is unclear whether people PD with and without freezing of gait differed in bouts without a freezing episode. The relatively small number of people with $\mathrm{PD}$ who exhibited freezing of gait in our sample prevented a sub-analysis examining this question. However, future studies may investigate whether freezing of gait is similarly associated with phase-dependent DT costs.

Overall, these results challenge our understanding of DT costs while walking, particularly in people with PD. With growing evidence that cortical control occurs during specific phases of gait, it is necessary to re-evaluate traditional concepts of DT interference that may neglect the phasic structure of control during locomotion. Our results suggest that PD-related DT interference occurs only immediately before and during postural adjustments at weight transfer. Interventions, particularly those utilizing DT and multi-task training paradigms, may benefit from focusing on postural adjustments during gait, and future research should directly examine this question using mobile neuroimaging modalities time-locked to phases of the gait cycle.

\section{ETHICS STATEMENT}

This study was carried out in accordance with the recommendations of the Oregon Health \& Science University (OHSU) and Veterans Affairs Portland Health Care System (VAPORHCS) joint institutional review board (IRB) with written informed consent from all subjects. All subjects gave written informed consent in accordance with the Declaration of Helsinki. The protocol was approved by the OHSU (\#4131) and the OHSU/VAPORHCS joint IRB (\#8979).

\section{AUTHOR CONTRIBUTIONS}

PF, MM, and FH conceptualized the question and hypothesis. $\mathrm{FH}, \mathrm{JN}$, and MM designed the study from which the data originates. PF, MM, and CC contributed to data collection and analysis. $\mathrm{PF}, \mathrm{MM}, \mathrm{CC}, \mathrm{JN}$, and $\mathrm{FH}$ contributed to the interpretation, writing and editing of the manuscript. PF wrote the first draft.

\section{ACKNOWLEDGMENTS}

The authors would like to thank Graham Harker, Natassja Pal, Michael Fleming, Heather Schlueter, and Peter Martin for their assistance during data collection and subject recruitment, and special thanks to Dr. Patty Carlson-Kuhta for her contributions throughout the study. This study was supported through funding from the NIH 2R01AG006457 (FH), R00HD078492 01A1 (MM), and VA Merit 5I01RX001075 (FH). 


\section{REFERENCES}

1. Morris ME, Iansek R, Matyas TA, Summers JJ. The pathogenesis of gait hypokinesia in Parkinson's disease. Brain (1994) 117(Pt 5):1169-81. doi:10.1093/ brain/117.5.1169

2. Hanakawa T, Katsumi Y, Fukuyama H, Honda M, Hayashi T, Kimura J, et al. Mechanisms underlying gait disturbance in Parkinson's disease: a single photon emission computed tomography study. Brain (1999) 122(Pt 7):1271-82. doi:10.1093/brain/122.7.1271

3. Braak H, Del Tredici K, Rub U, De Vos RA, Jansen Steur EN, Braak E. Staging of brain pathology related to sporadic Parkinson's disease. Neurobiol Aging (2003) 24:197-211. doi:10.1016/S0197-4580(02)00065-9

4. Bohnen NI, Albin RL. The cholinergic system and Parkinson disease. Behav Brain Res (2011) 221:564-73. doi:10.1016/j.bbr.2009.12.048

5. Galna B, Lord S, Burn DJ, Rochester L. Progression of gait dysfunction in incident Parkinson's disease: impact of medication and phenotype. Mov Disord (2015) 30:359-67. doi:10.1002/mds.26110

6. Bohnen NI, Jahn K. Imaging: what can it tell us about parkinsonian gait? Mov Disord (2013) 28:1492-500. doi:10.1002/mds.25534

7. Wu T, Hallett M, Chan P. Motor automaticity in Parkinson's disease. Neurobiol Dis (2015) 82:226-34. doi:10.1016/j.nbd.2015.06.014

8. Gilat M, Bell PT, Ehgoetz Martens KA, Georgiades MJ, Hall JM, Walton CC, et al. Dopamine depletion impairs gait automaticity by altering cortico-striatal and cerebellar processing in Parkinson's disease. Neuroimage (2017) 152:207-20. doi:10.1016/j.neuroimage.2017.02.073

9. Maidan I, Nieuwhof F, Bernad-Elazari H, Reelick MF, Bloem BR, Giladi N, et al. The role of the frontal lobe in complex walking among patients with Parkinson's disease and healthy older adults: an fNIRS study. Neurorehabil Neural Repair (2016) 30:963-71. doi:10.1177/1545968316650426

10. Hausdorff JM, Cudkowicz ME, Firtion R, Wei JY, Goldberger AL. Gait variability and basal ganglia disorders: stride-to-stride variations of gait cycle timing in Parkinson's disease and Huntington's disease. Mov Disord (1998) 13:428-37. doi:10.1002/mds.870130310

11. Hausdorff JM, Balash J, Giladi N. Effects of cognitive challenge on gait variability in patients with Parkinson's disease. J Geriatr Psychiatry Neurol (2003) 16:53-8. doi:10.1177/0891988702250580

12. Schaafsma JD, Giladi N, Balash Y, Bartels AL, Gurevich T, Hausdorff JM. Gait dynamics in Parkinson's disease: relationship to Parkinsonian features, falls and response to levodopa. J Neurol Sci (2003) 212:47-53. doi:10.1016/ S0022-510X(03)00104-7

13. Moon Y, Sung J, An R, Hernandez ME, Sosnoff JJ. Gait variability in people with neurological disorders: a systematic review and meta-analysis. Hum Mov Sci (2016) 47:197-208. doi:10.1016/j.humov.2016.03.010

14. Hausdorff JM. Gait dynamics in Parkinson's disease: common and distinct behavior among stride length, gait variability, and fractal-like scaling. Chaos (2009) 19:026113. doi:10.1063/1.3147408

15. Morris ME, Iansek R, Matyas TA, Summers JJ. Stride length regulation in Parkinson's disease. Normalization strategies and underlying mechanisms. Brain (1996) 119(Pt 2):551-68. doi:10.1093/brain/119.2.551

16. Wu T, Kansaku K, Hallett M. How self-initiated memorized movements become automatic: a functional MRI study. J Neurophysiol (2004) 91:1690-8. doi:10.1152/jn.01052.2003

17. Clark DJ. Automaticity of walking: functional significance, mechanisms, measurement and rehabilitation strategies. Front Hum Neurosci (2015) 9:246. doi:10.3389/fnhum.2015.00246

18. O'Shea S, Morris ME, Iansek R. Dual task interference during gait in people with Parkinson disease: effects of motor versus cognitive secondary tasks. Phys Ther (2002) 82:888-97.

19. Rochester L, Hetherington V, Jones D, Nieuwboer A, Willems AM, Kwakkel G, et al. Attending to the task: interference effects of functional tasks on walking in Parkinson's disease and the roles of cognition, depression, fatigue, and balance. Arch Phys Med Rehabil (2004) 85:1578-85. doi:10.1016/j.apmr.2004.01.025

20. Rochester L, Nieuwboer A, Baker K, Hetherington V, Willems AM, Kwakkel G, et al. Walking speed during single and dual tasks in Parkinson's disease: which characteristics are important? Mov Disord (2008) 23:2312-8. doi:10.1002/ mds. 22219

21. Pashler H. Dual-task interference in simple tasks: data and theory. Psychol Bull (1994) 116:220-44. doi:10.1037/0033-2909.116.2.220
22. Tombu M, Jolicoeur P. A central capacity sharing model of dual-task performance. J Exp Psychol Hum Percept Perform (2003) 29:3-18. doi:10.1037/ 0096-1523.29.1.3

23. Rochester L, Galna B, Lord S, Burn D. The nature of dual-task interference during gait in incident Parkinson's disease. Neuroscience (2014) 265:83-94. doi:10.1016/j.neuroscience.2014.01.041

24. Peterson DS, Horak FB. Neural control of walking in people with Parkinsonism. Physiology (Bethesda) (2016) 31:95-107. doi:10.1152/physiol.00034.2015

25. Kelly VE, Eusterbrock AJ, Shumway-Cook A. A review of dual-task walking deficits in people with Parkinson's disease: motor and cognitive contributions, mechanisms, and clinical implications. Parkinsons Dis (2012) 2012:918719. doi: $10.1155 / 2012 / 918719$

26. Lord S, Rochester L, Hetherington V, Allcock LM, Burn D. Executive dysfunction and attention contribute to gait interference in 'off' state Parkinson's disease. Gait Posture (2010) 31:169-74. doi:10.1016/j.gaitpost.2009.09.019

27. Peterson DS, Fling BW, Mancini M, Cohen RG, Nutt JG, Horak FB. Dual-task interference and brain structural connectivity in people with Parkinson's disease who freeze. J Neurol Neurosurg Psychiatry (2015) 86:786-92. doi:10.1136/ jnnp-2014-308840

28. de Souza Fortaleza AC, Mancini M, Carlson-Kuhta P, King LA, Nutt JG, Chagas EF, et al. Dual task interference on postural sway, postural transitions and gait in people with Parkinson's disease and freezing of gait. Gait Posture (2017) 56:76-81. doi:10.1016/j.gaitpost.2017.05.006

29. Gwin JT, Gramann K, Makeig S, Ferris DP. Electrocortical activity is coupled to gait cycle phase during treadmill walking. Neuroimage (2011) 54:1289-96. doi:10.1016/j.neuroimage.2010.08.066

30. Bruijn SM, Van Dieen JH, Daffertshofer A. Beta activity in the premotor cortex is increased during stabilized as compared to normal walking. Front Hum Neurosci (2015) 9:593. doi:10.3389/fnhum.2015.00593

31. Wagner J, Makeig S, Gola M, Neuper C, Muller-Putz G. Distinct beta band oscillatory networks subserving motor and cognitive control during gait adaptation. J Neurosci (2016) 36:2212-26. doi:10.1523/JNEUROSCI.3543-15.2016

32. Ihlen EAF, Goihl T, Wik PB, Sletvold O, Helbostad J, Vereijken B. Phasedependent changes in local dynamic stability of human gait. J Biomech (2012) 45:2208-14. doi:10.1016/j.jbiomech.2012.06.022

33. Ihlen EAF, Sletvold O, Goihl T, Wik PB, Vereijken B, Helbostad J. Older adults have unstable gait kinematics during weight transfer. J Biomech (2012) 45:1559-65. doi:10.1016/j.jbiomech.2012.04.021

34. Ihlen EA, Weiss A, Helbostad JL, Hausdorff JM. The discriminant value of phase-dependent local dynamic stability of daily life walking in older adult community-dwelling fallers and nonfallers. Biomed Res Int (2015) 2015:402596. doi:10.1155/2015/402596

35. Ihlen EA, Weiss A, Beck Y, Helbostad JL, Hausdorff JM. A comparison study of local dynamic stability measures of daily life walking in older adult community-dwelling fallers and non-fallers. J Biomech (2016) 49(9):1498-503. doi:10.1016/j.jbiomech.2016.03.019

36. Gelb DJ, Oliver E, Gilman S. Diagnostic criteria for Parkinson disease. Arch Neurol (1999) 56:33-9. doi:10.1001/archneur.56.1.33

37. Goetz CG, Tilley BC, Shaftman SR, Stebbins GT, Fahn S, Martinez-Martin P, et al. Movement Disorder Society-sponsored revision of the Unified Parkinson's Disease Rating Scale (MDS-UPDRS): scale presentation and clinimetric testing results. Mov Disord (2008) 23:2129-70. doi:10.1002/mds.22340

38. Stebbins GT, Goetz CG, Burn DJ, Jankovic J, Khoo TK, Tilley BC. How to identify tremor dominant and postural instability/gait difficulty groups with the movement disorder society unified Parkinson's disease rating scale: comparison with the unified Parkinson's disease rating scale. Mov Disord (2013) 28:668-70. doi:10.1002/mds.25383

39. Nasreddine ZS, Phillips NA, Bedirian V, Charbonneau S, Whitehead V, Collin I, et al. The Montreal Cognitive Assessment, MoCA: a brief screening tool for mild cognitive impairment. J Am Geriatr Soc (2005) 53:695-9. doi:10.1111/j.1532-5415.2005.53221.x

40. El-Gohary M, Pearson S, Mcnames J, Mancini M, Horak F, Mellone S, et al. Continuous monitoring of turning in patients with movement disability. Sensors (Basel) (2013) 14:356-69. doi:10.3390/s140100356

41. Salarian A, Russmann H, Vingerhoets FJ, Dehollain C, Blanc Y, Burkhard PR, et al. Gait assessment in Parkinson's disease: toward an ambulatory system for long-term monitoring. IEEE Trans Biomed Eng (2004) 51:1434-43. doi:10.1109/TBME.2004.827933 
42. Kantz H. A robust method to estimate the maximal Lyapunov exponent of a time series. Phys Lett A (1994) 185:77-87. doi:10.1016/0375-9601(94)90991-1

43. Sloot LH, Van Schooten KS, Bruijn SM, Kingma H, Pijnappels M, Van Dieën JH. Sensitivity of local dynamic stability of over-ground walking to balance impairment due to galvanic vestibular stimulation. Ann Biomed Eng (2011) 39:1563-9. doi:10.1007/s10439-010-0240-y

44. van Schooten KS, Rispens SM, Elders PJ, Van Dieen JH, Pijnappels M. Toward ambulatory balance assessment: estimating variability and stability from short bouts of gait. Gait Posture (2014) 39:695-9. doi:10.1016/j.gaitpost. 2013.09.020

45. Fino PC. A preliminary study of longitudinal differences in local dynamic stability between recently concussed and healthy athletes during single and dualtask gait. J Biomech (2016) 49:1983-8. doi:10.1016/j.jbiomech.2016.05.004

46. Dingwell JB, Cusumano JP, Cavanagh PR, Sternad D. Local dynamic stability versus kinematic variability of continuous overground and treadmill walking. J Biomech Eng (2001) 123:27-32. doi:10.1115/1.1336798

47. Bruijn S, Meijer O, Beek P, Van Dieën J. Assessing the stability of human locomotion: a review of current measures. J R Soc Interface (2013) 10:20120999. doi:10.1098/rsif.2012.0999

48. Hak L, Houdijk H, Beek PJ, Van Dieen JH. Steps to take to enhance gait stability: the effect of stride frequency, stride length, and walking speed on local dynamic stability and margins of stability. PLoS One (2013) 8:e82842. doi:10.1371/journal.pone.0082842

49. Terrier P, Reynard F. Effect of age on the variability and stability of gait: a cross-sectional treadmill study in healthy individuals between 20 and 69 years of age. Gait Posture (2015) 41:170-4. doi:10.1016/j.gaitpost.2014.09.024

50. Seeber M, Scherer R, Wagner J, Solis-Escalante T, Muller-Putz GR. EEG beta suppression and low gamma modulation are different elements of human upright walking. Front Hum Neurosci (2014) 8:485. doi:10.3389/ fnhum.2014.00485

51. Bulea TC, Kim J, Damiano DL, Stanley CJ, Park HS. Prefrontal, posterior parietal and sensorimotor network activity underlying speed control during walking. Front Hum Neurosci (2015) 9:247. doi:10.3389/fnhum.2015.00247

52. Seeber M, Scherer R, Wagner J, Solis-Escalante T, Muller-Putz GR. High and low gamma EEG oscillations in central sensorimotor areas are conversely modulated during the human gait cycle. Neuroimage (2015) 112:318-26. doi:10.1016/j.neuroimage.2015.03.045

53. Wagner J, Solis-Escalante T, Grieshofer P, Neuper C, Muller-Putz G, Scherer R. Level of participation in robotic-assisted treadmill walking modulates midline sensorimotor EEG rhythms in able-bodied subjects. Neuroimage (2012) 63:1203-11. doi:10.1016/j.neuroimage.2012.08.019

54. Bent LR, Inglis JT, Mcfadyen BJ. When is vestibular information important during walking? J Neurophysiol (2004) 92:1269-75. doi:10.1152/jn.01260.2003

55. Bauby CE, Kuo AD. Active control of lateral balance in human walking. J Biomech (2000) 33:1433-40. doi:10.1016/S0021-9290(00)00101-9

56. O'Connor SM, Kuo AD. Direction-dependent control of balance during walking and standing. J Neurophysiol (2009) 102:1411-9. doi:10.1152/jn. 00131.2009

57. Townsend MA. Biped gait stabilization via foot placement. J Biomech (1985) 18:21-38. doi:10.1016/0021-9290(85)90042-9
58. Hof AL, Gazendam MG, Sinke WE. The condition for dynamic stability. J Biomech (2005) 38:1-8. doi:10.1016/j.jbiomech.2004.03.025

59. Wang Y, Srinivasan M. Stepping in the direction of the fall: the next foot placement can be predicted from current upper body state in steady-state walking. Biol Lett (2014) 10:20140405. doi:10.1098/rsbl.2014.0405

60. Rosin R, Topka H, Dichgans J. Gait initiation in Parkinson's disease. Mov Disord (1997) 12:682-90. doi:10.1002/mds.870120509

61. Hanakawa T. Neuroimaging of standing and walking: special emphasis on Parkinsonian gait. Parkinsonism Relat Disord (2006) 12:S70-5. doi:10.1016/j. parkreldis.2006.05.009

62. Gallea C, Ewenczyk C, Degos B, Welter ML, Grabli D, Leu-Semenescu S, et al. Pedunculopontine network dysfunction in Parkinson's disease with postural control and sleep disorders. Mov Disord (2017) 32:693-704. doi:10.1002/ mds. 26923

63. Beauchet O, Dubost V, Aminian K, Gonthier R, Kressig RW. Dual-task-related gait changes in the elderly: does the type of cognitive task matter? J Mot Behav (2005) 37:259-64.

64. Hausdorff JM, Schweiger A, Herman T, Yogev-Seligmann G, Giladi N. Dual-task decrements in gait: contributing factors among healthy older adults. J Gerontol A Biol Sci Med Sci (2008) 63:1335-43. doi:10.1093/gerona/ 63.12.1335

65. Montero-Odasso M, Muir SW, Speechley M. Dual-task complexity affects gait in people with mild cognitive impairment: the interplay between gait variability, dual tasking, and risk of falls. Arch Phys Med Rehabil (2012) 93:293-9. doi:10.1016/j.apmr.2011.08.026

66. Lajoie Y, Teasdale N, Bard C, Fleury M. Attentional demands for static and dynamic equilibrium. Exp Brain Res (1993) 97:139-44. doi:10.1007/ BF00228824

67. Smulders K, Esselink RA, Weiss A, Kessels RP, Geurts AC, Bloem BR Assessment of dual tasking has no clinical value for fall prediction in Parkinson's disease. J Neurol (2012) 259:1840-7. doi:10.1007/s00415-012-6419-4

68. Menant JC, Schoene D, Sarofim M, Lord SR. Single and dual task tests of gait speed are equivalent in the prediction of falls in older people: a systematic review and meta-analysis. Ageing Res Rev (2014) 16:83-104. doi:10.1016/j. arr.2014.06.001

Conflict of Interest Statement: FBH has a significant financial interest in APDM, a company that may have a commercial interest in the results of this research and technology. This potential conflict has been reviewed and managed by OHSU. All other authors declare that the research was conducted in the absence of any commercial or financial relationships that could be construed as a potential conflict of interest.

Copyright (c) 2018 Fino, Mancini, Curtze, Nutt and Horak. This is an open-access article distributed under the terms of the Creative Commons Attribution License (CC BY). The use, distribution or reproduction in other forums is permitted, provided the original author(s) and the copyright owner are credited and that the original publication in this journal is cited, in accordance with accepted academic practice. No use, distribution or reproduction is permitted which does not comply with these terms. 